\title{
RESILIENCIA DE LA COMUNIDAD FITOPLANCTÓNICA EN LA LAGUNA ANDINA DE PAPALLACTA Y SUS AFLUENTES, OCHO AÑOS DESPUÉS DE UN DERRAME PETROLERO
}

\author{
RESILIENCE OF PHYTOPLANKTON COMMUNITY IN THE ANDEAN PAPALLACTA \\ LAGOON AND ITS TRIBUTARIES, EIGHT YEARS AFTER AN OIL SPILL
}

\author{
Marcos Molina-Santos ${ }^{1}$, Esteban Terneus-Jácome ${ }^{1}$, Patricio Yánez-Moretta ${ }^{2}$, \\ Mario Cueva-Sánchez ${ }^{1}$
}

\footnotetext{
${ }^{1}$ Escuela de Biología Aplicada, Universidad Internacional del Ecuador, Av. Simón Bolívar y Jorge Fernández s/n, Quito, Ecuador.

${ }^{2}$ Escuela de Gestión Turística y Medio Ambiente, Universidad Internacional del Ecuador, Av. Simón Bolívar y Jorge Fernández $s / n$, Quito, Ecuador.
}

*Autor para correspondencia: apyanez@hotmail.com

\begin{abstract}
Resumen
En abril de 2003 se produjo un derrame de crudo en la zona de Papallacta (región andina norte de Ecuador), luego del desastre se realizaron labores de biorremediación tanto en la laguna como en sus afluentes. El presente trabajo abordó la comunidad fitoplanctónica como bioindicadora de la calidad actual del cuerpo de agua, considerando 28 sitios en ríos afluentes y en la laguna, tanto en época seca, como en la de transición y lluviosa, desde diciembre 2010 hasta julio 2011. Se identificaron y contabilizaron los géneros de fitoplancton encontrados en cada sitio, abordando paralelalmente la historia natural de cada grupo de algas. Los géneros pertenecieron a tres grupos: diatomeas, cianobacterias y algas verdes; el primero es el grupo con mayor distribución y con los valores de riqueza y abundancia más altos. Las correlaciones entre géneros y variables abióticas presentaron diferencias relacionadas con la estacionalidad. Sin embargo, se observó la constante presencia de géneros indicadores de alta carga orgánica como Synedra y Oscillatoria. Se aporta también información acerca de los géneros que ahora predominan tanto en la laguna como en sus afluentes, y las características que influyen en su distribución y que permiten determinar el estado de salud ecológica de la Laguna de Papallacta, que ahora se la puede catalogar como mesotrófica. La presencia actual de crudo del derrame se concentra en puntos específicos en el fondo de la laguna, permitiendo que la productividad primaria en la zona fótica se desarrolle con normalidad.
\end{abstract}

Palabras claves: limnología, lagunas andinas, derrame petrolero, fitoplancton, bioindicador, calidad del agua. 


\begin{abstract}
In April 2003, there was an oil spill in the Papallacta zone (Ecuador: northern Andes), after the disaster bioremediation work was carried out in the lagoon and in its tributaries. We analyze the phytoplankton community as bioindicator of the current quality of the water body, considering 28 places in tributaries and in the lagoon, both in the dry season, transitional and rainy season, from December 2010 to July 2011, we identified and counted the genera of phytoplankton found in each sampling point, also considering the natural history of each group of algae. Diatoms, cyanobacteria and green algae were the major groups, the first of them was the most widely distributed with higher values in richness and abundance. The correlations between genera and abiotic variables showed differences related to seasonality. However, there was the constant presence of certain genera as Synedra and Oscillatoria, high organic load indicators. This research also provides information about the genera that now dominate both in the lagoon and its tributaries and the characteristics that influence their distribution and allow to determine the ecological health of Laguna de Papallacta, now it can be classified as mesotrophic. The presence of oil is restricted on specific points on the bottom of the lagoon, allowing that primary productivity in the photic zone develops normally.

Keywords: limnology, Andean lagoons, oil spill, phytoplankton, bioindicator, water quality.

Forma sugerida de citar: Molina-Santos, M., Terneus-Jácome, E., Yánez-Moretta, P. y Cueva-Sánchez, M. 2018. Resiliencia de la comunidad fitoplanctónica en la laguna andina de Papallacta y sus afluentes, ocho años después de un derrame petrolero. La Granja: Revista de Ciencias de la Vida. Vol. 28(2):67-83. http:/ / doi.org/10.17163/lgr.n28.2018.05.
\end{abstract}




\section{Introducción}

El número de especies de fitoplancton en el mundo es aún desconocido, en el océano se estiman unas 5000, número que puede ser mayor en ecosistemas acuáticos continentales como lagunas, ya que no forman un solo cuerpo de agua (Molina Santos, 2013), siendo más bien sistemas (río-laguna) relativamente aislados uno del otro, a diferencia del océano que es un ambiente con un área mayor pero con características relativamente similares. Es así como lagunas y ríos suelen estar influenciados por cambios estacionales y variables abióticas inconstantes, que pueden ser determinantes para la diversidad del fitoplancton (O'Sullivan y Reynolds, 2004; Escobar, Terneus y Yánez, 2013).

La calidad y cantidad del fitoplancton en un cuerpo de agua permiten inferir atributos del estado del hábitat, tales como el balance de nutrientes, disponibilidad de alimentos para heterótrofos menores, calidad de la luz, movimientos del agua, entre otras. Estas características hacen que el estudio de sus poblaciones sea fundamental para comprender el estado de un sistema hídrico (O'Sullivan y Reynolds, 2004).

Varios factores causan estrés sobre el fitoplancton y sus comunidades, entre ellos la radiación ultravioleta, acidificación, eutrofización y el calentamiento global, los cuales pueden actuar modificando la estructura y dinámica de tales comunidades (Delgado-Molina et al., 2009; Belinger y Sigee, 2010).

\subsection{El fitoplancton como bioindicador}

El fitoplancton suele presentar cierta tolerancia a la contaminación orgánica y permite conocer los cambios que se presentan en cuerpos de agua en los que ha ocurrido contaminación (Arce et al., 2006), este evento incluso puede ayudar en la determinación de especies capaces de degradar materia orgánica o depurar el ambiente a través de la fotosíntesis, la cual agrega oxígeno disuelto al sistema, útil para otros organismos y para la oxidación de dicha materia (Arce et al., 2006; Suthers y Rissik, 2009; Terneus, 2018).

Por otra parte, el fitoplancton suele ser el primer grupo afectado por metales pesados, esto desencadena un problema no solamente para él sino también para los grupos superiores debido a que al absorber los metales a nivel celular, desencadena una biomagnificación en los herbívoros de primer orden que lo consumen y luego en los carnívoros que consumen a éstos y así sucesivamente (Bahnasawy, Khidr y Dheina, 2011; González-Dávila, 1995).

El nivel de afectación que puede mostrar el fitoplancton depende de factores como concentración de oxígeno, temperatura, $\mathrm{pH}$ y salinidad del agua, ya que de ella obtiene sus nutrientes; por lo que el agua puede ser considerada la principal fuente de contaminación del fitoplancton (Bahnasawy, Khidr y Dheina, 2011).

\subsection{El derrame de petróleo en Papallacta y los objetivos de la presente investiga- ción}

A las 4 h00 del 8 de abril de 2003 se produjo un derrame de crudo en la zona altoandina de Papallacta, generado por una ruptura de la tubería del Sistema del Oleoducto Transecuatoriano (SOTE), manejado por Petroecuador; tal evento provocó una afectación sobre los cauces de los ríos Cachalarca, Tambo y Sucus (PetroEcuador, 2006), así como en la Laguna de Papallacta (Figura 1). El sitio del derrame fue en el sector conocido como El Guango a 3631 msnm (Armisen, Cruz y Larrosa, 2005), coordenadas UTM N 9959586 y E 812820 (WGS84), cerca al Río Sucus. 


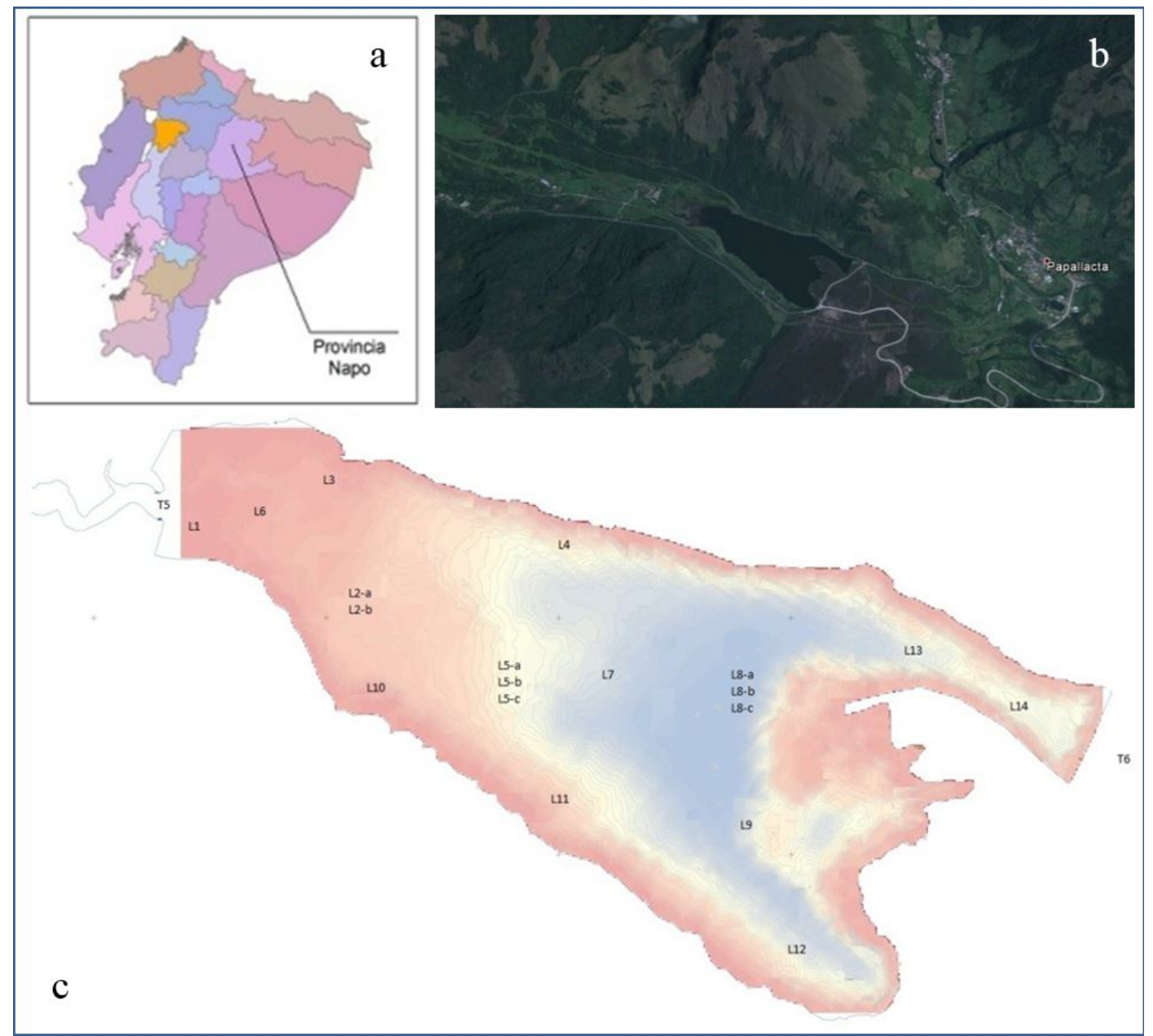

Figura 1. Situación geográfica de la Laguna de Papallacta y los sitios de muestreo: a. Napo, provincia andino-amazónica, en relación al Ecuador continental; b. Imagen satelital de la laguna (Google Earth, enero 2013); c. Ubicación de puntos de muestreo en diferentes zonas de la laguna.

Se derramaron 22000 barriles; una parte de este petróleo se disolvió en el agua, produciéndose un flujo continuo por los Ríos Tambo y Sucus hacia la laguna. En principio se pudo ver una capa de petróleo de entre 0,1 y 70,0 cm de espesor en una superficie aproximada de 6,5 hectáreas de la laguna; luego por procesos naturales, el crudo se precipitó al fondo (Pino et al., 2005). Se pudo observar que cerca al sitio del derrame el suelo estaba cubierto con una capa de petróleo de seis centímetros de espesor, afectando no solamente el agua sino también la flora y la fauna del lugar (Armisen, Cruz y Larrosa, 2005; Molina Santos, 2013; PetroEcuador, 2006).

Petroecuador puso en marcha el plan de contingencias para detener los efectos sobre el ecosistema; luego de la evaluación correspondiente, se empezó con el programa de biorremediación, consistente en el desarrollo de trabajos en el sitio de la rotura, las riberas de los ríos Sucus y Tambo, agua y suelo contaminados al borde y en la misma laguna, todo como parte del proceso de restauración de los ecosistemas afectados (PetroEcuador, 2006; Molina Santos, 2013).

Dentro de este contexto, se planteó la presente investigación buscando:

- Determinar la composición de la comunidad de fitoplancton en los ecosistemas acuáticos en la zona afectada por el derrame petrolero.

- Categorizar los grupos de fitoplancton en función de sus características de bioindicación. 
- Evaluar el estado actual de salud ecológica de la Laguna de Papallacta, considerando la comunidad fitoplanctónica como bioindicador.

\section{Materiales y métodos}

\section{1 Área de estudio}

La Laguna de Papallacta (Figura 1) y sus afluentes, los ríos Tambo y Sucus, se encuentran en la provincia de Napo, en un valle glaciar a $70 \mathrm{~km}$ al este de la ciudad de Quito, en el extremo suroccidental del Parque Nacional Cayambe-Coca, a una altitud aproximada de $3350 \mathrm{msnm}$.

Las temperaturas medias locales oscilan entre 6 ${ }^{\circ} \mathrm{C}$ a $8{ }^{\circ} \mathrm{C}$; la precipitación media anual entre 1250 a $1400 \mathrm{~mm}$ /año (PRAA, 2007).

Los sitios de recolección de las muestras fueron ubicados en varias zonas, desde poco antes del sitio del derrame (UTM E 812 856, N 9959 639) hasta un punto en el efluente de la laguna (UTM E 816 860, N 9958 048). Se establecieron 28 puntos de muestro (19 en laguna y 9 en río), las muestras se tomaron considerando las condiciones físicas del ecosistema; por esto se usaron dos métodos diferentes de acuerdo al sitio. En la laguna se colectó desde un bote, mientras que las muestras de río se colectaron directamente del sustrato. Los puntos de laguna se identifican con la letra L y los puntos de río con la letra $\mathrm{T}$ (Figura 1).

Los puntos de muestreo en la laguna se establecieron con base en un análisis batimétrico previo, de esta manera se determinaron las profundidades de cada punto y su topografía con mejor precisión, lo que ayudó a identificar los sitios donde puede acumularse el crudo hacia el fondo.

El sector de Papallacta posee características de páramo herbáceo, siendo dominantes en el paisaje los pajonales de Calamagrostis sp. y Stipa sp., así como el musgo; eventualmente se desarrollan árboles y arbustos de crecimiento irregular de los géneros Oreopanax, Gynoxys, Axinaea, Brachyotum, Hypericum, Buddleja, Polylepis (Yánez, 2014).

La diversidad de peces es baja en los páramos de la zona de estudio, se destacan las truchas (Oncorhynchus mykiss) (Molina Santos, 2013) como elementos introducidos y utilizadas para alimento y con fines comerciales.

\subsection{Métodos}

Los muestreos se llevaron a cabo en períodos correspondientes a tres épocas del año: seca (diciembre 2010), transición (marzo 2011) y lluviosa (julio 2011). El análisis estacional suele ser muy importante, ya que los cambios influyen en los patrones de vida de los organismos fitoplanctónicos cuando cambia la temperatura del agua y su movimiento, disponibilidad de luz, prinipalmente (Marshall, 1965).

En la laguna se realizó un muestreo aleatorio estratificado con una Botella Van Dorn, la cual desciende verticalmente hasta la profundidad deseada. La botella recogió dos litros de agua a la vez, que luego fueron filtrados a través de una red de fitoplancton de 30 micras con un cubilete enroscado al extremo, este proceso permitió recoger la mayor cantidad de plancton, facilitando el análisis posterior en laboratorio. Para cada sitio se tomaron tres submuestras entre 1,0 a 1,5 m de profundidad. Los especímenes fueron preservados en formalina al $4 \%$ y almacenados en envases de color ámbar.

En los ríos se utilizó una red Surber con ojo de 85 micras, debido a que el fitoplancton se presenta de otra manera en este ecosistema, ya que suele estar pegado a las rocas o plantas de ribera en forma de perifiton y epiliton; la profundidad del río no sobrepasó los 0,8 $\mathrm{m}$ (profundidad más alta en época lluviosa), de esta manera se tomaron muestras hasta a $0,2 \mathrm{~m}$ de profundidad. La red utilizada consiste de dos marcos cada uno de $30 \mathrm{~cm} \times 30 \mathrm{~cm}$, unidos por abrazaderas, un marco se coloca sobre la superficie a muestrear y una red de $60 \mathrm{~cm}$ unida al segundo marco permite recoger la muestra (Alberta Environment, 2006). Con esta herramienta se recogió el perifiton pegado a las piedras principalmente y en ciertos casos también en las orillas.

Tanto en río como en laguna se tomaron datos in situ, con un medidor de oxígeno y un multiparámetros digital: $\mathrm{pH}$, conductividad, sólidos suspendidos, oxígeno disuelto, temperatura del agua, profundidad total del punto y profundidad del disco Secchi. Adicionalmente, se enviaron muestras de cada sitio a un laboratorio para análisis de Demanda Bioquímica de Oxígeno (DBO), Demanda Química de Oxígeno (DQO), cromo, bario, plomo, vanadio, fenoles, nitrógeno global, sólidos disueltos, turbidez e Hidrocarburos Totales de Petróleo (TPH). 


\subsection{Análisis de muestras en laboratorio}

Se utilizaron dos técnicas, la primera consistió en utilizar embudos de separación de $250 \mathrm{ml}$ y $500 \mathrm{ml}$, en los que se dejó la muestra en decantación entre 24 y 72 horas para separar el fitoplancton de los sólidos en suspensión, luego se dejó caer solamente el sedimento separado, el cual se mantuvo en tubos de ensayo; esta técnica es viable cuando se trata de muestras de río porque tienen mayor carga de sedimento que debe ser decantado. La segunda técnica fue la centrifugación, que se utilizó cuando existían bajas concentraciones de fitoplancton, para esto se tomaron $200 \mathrm{ml}$ de muestra homogenizada y se centrifugó a baja velocidad durante 20 minutos, de esta manera se pueden procesar más muestras en menor tiempo.

La identificación de fitoplancton se realizó en el Laboratorio de la Universidad Internacional del Ecuador. Para el conteo e identificación se utilizaron microscopios Olympus CX21FS1. Cada muestra observada tuvo el mismo volumen, para esto se colocaron tres gotas de $100 \mu \mathrm{l}$ de cada muestra en una placa cóncava con varias hendiduras, esto permite realizar un conteo relativamente rápido. Cada gota fue analizada minuciosamente, para esto la gota se dividió en nueve partes con una cuadricula, así se obtuvo el número real de géneros e individuos por gota. El resultado final del conteo fue expresado en número de colonias por mililitro (NCM).

Para la identificación taxonómica se utilizaron las claves de (Belinger y Sigee, 2010; Canter-Lund, 2012; California Academy of Sciences, 2012; Fourtanier y Kociolek, 2009).

\subsection{Análisis de información}

Los sitios de muestreo, en función de sus características abióticas, fueron sometidos a un análisis de similitud (Cluster Analysis) (Hill, Lewicki y Lewicki,
2006), basado en distancias euclidianas entre sitios de acuerdo a lo recomendado por (Yánez, 2005).

Para el análisis de la biota fitoplanctónica se aplicó el Índice de tolerancia a contaminación orgánica de Palmer, en el que se proponen 20 géneros como los más tolerantes a este tipo de contaminación y se les asigna un valor de tolerancia. Para caracterizar a un sitio los índices individuales se suman $y$, de acuerdo al valor total obtenido, se determina qué tanta carga orgánica hay en el sitio. Palmer determinó que un valor de Índice por sitio superior a 20 indica una alta carga orgánica, un resultado entre 15 y 19 indica una evidencia probable de alta contaminación orgánica y valores inferiores pueden indicar poca carga orgánica, muestra no representativa o que ciertos factores particulares están influyendo sobre la presencia de fitoplancton (Hern et al., 1979).

Para comparar los sitios en función de su composición fitoplanctónica, se utilizó el Índice de similitud de Steinhaus (Bray-Curtis), recomendado por (Goslee, Urban et al., 2007; Yoshioka, 2008), con el cual a su vez se crearon los Cladogramas respectivos.

Finalmente, se realizó un Análisis Canónico de Correspondencias para identificar la relación entre las variables abióticas con respecto a la composición de la comunidad biológica de interés en los sitios (Ter Braak y Verdonschot, 1995). Para este análisis se tomaron en cuenta solo los géneros con mayor abundancia.

\section{Resultados y discusión}

\subsection{Grupos de fitoplancton registrados}

Se encontraron tres grandes grupos de fitoplancton (en la Laguna de Papallacta y tributarios Tambo y Sucus): Diatomeas (Bacillariophyta), algas verdeazules o cianobacterias (Cyanophyta) y algas verdes (Chlorophyta), distribuidos en 21 géneros (Tabla 1). 
Tabla 1. Géneros de microalgas registrados en Papallacta en afluentes y laguna (se muestran las abundancias totales estimadas por época de muestreo y la abundancia total en número de colonias por mililitro $=\mathrm{NCM}$ )

\begin{tabular}{|c|c|c|c|c|c|c|c|}
\hline Grupo & Clasificación & No. & Género & Época seca & $\begin{array}{l}\text { Época de } \\
\text { transición }\end{array}$ & $\begin{array}{l}\text { Época de } \\
\text { lluvia }\end{array}$ & NCM Total \\
\hline \multirow{14}{*}{ Diatomeas } & \multirow[b]{2}{*}{ Centrales } & 1 & Cyclotella & 3000 & 9000 & 0 & 12000 \\
\hline & & 2 & Melosira & 5415000 & 1508000 & 194000 & 7117000 \\
\hline & \multirow{12}{*}{ Penales } & 3 & Campylodiscus & 72000 & 0 & 0 & 72000 \\
\hline & & 4 & Cymbella & 625000 & 374000 & 183000 & 1182000 \\
\hline & & 5 & Diploneis & 13000 & 12000 & 3000 & 28000 \\
\hline & & 6 & Frustulia & 1000 & 3000 & 0 & 4000 \\
\hline & & 7 & Hantzschia & 2742000 & 663000 & 289000 & 3694000 \\
\hline & & 8 & Navicula & 6092000 & 4121000 & 551000 & 10764000 \\
\hline & & 9 & Neidium & 5000 & 1000 & 6000 & 12000 \\
\hline & & 10 & Nitzschia & 8964500 & 1662000 & 353000 & 10979500 \\
\hline & & 11 & Pinnularia & 57000 & 0 & 0 & 57000 \\
\hline & & 12 & Rhopalodia & 49000 & 23000 & 0 & 72000 \\
\hline & & 13 & Surirella & 12000 & 2000 & 2000 & 16000 \\
\hline & & 14 & Synedra & 4215500 & 1255000 & 455000 & 5925500 \\
\hline \multirow{3}{*}{ Cianobacterias } & \multirow{3}{*}{ Cyanophyta } & 15 & Microcystis & 158000 & 16000 & 0 & 174000 \\
\hline & & 16 & Oscillatoria & 268000 & 138000 & 451000 & 857000 \\
\hline & & 17 & Spirulina & 0 & 0 & 3000 & 3000 \\
\hline \multirow{4}{*}{ Algas verdes } & \multirow{4}{*}{ Chlorophyta } & 18 & Ankistrodesmus & 1000 & 41000 & 9000 & 51000 \\
\hline & & 19 & Mougeotia & 95000 & 38000 & 0 & 133000 \\
\hline & & 20 & Rhizoclonium & 9000 & 4000 & 92000 & 105000 \\
\hline & & 21 & Spirogyra & 2000 & 0 & 0 & 2000 \\
\hline
\end{tabular}

\subsection{Análisis de clasificación de los sitios en función de sus características abióticas}

Durante la época seca se pudo distinguir tres tipos de sitios (Figura 2), signados con la letra L para los de laguna y T para los de Río; el más grande conformado por 21 sitios (L10 a L13), entre los que se encuentra $\mathrm{T} 5$ correspondiente a la desembocadura del río y T6 al punto de salida del efluente de la Laguna. Estos 21 sitios tienen mayor similitud debido a que los valores de conductividad, sólidos suspendidos y oxígeno disuelto son similares.

Otro grupo está conformado por tres puntos de río (T1A, T0 y T1B), con valores similares de conductividad, temperatura, oxígeno disuelto y sólidos suspendidos. El último grupo se conforma también por tres puntos de río (T3B, T2B y T3A), con valores de turbidez, conductividad, profundidad y $\mathrm{pH}$ similares. 


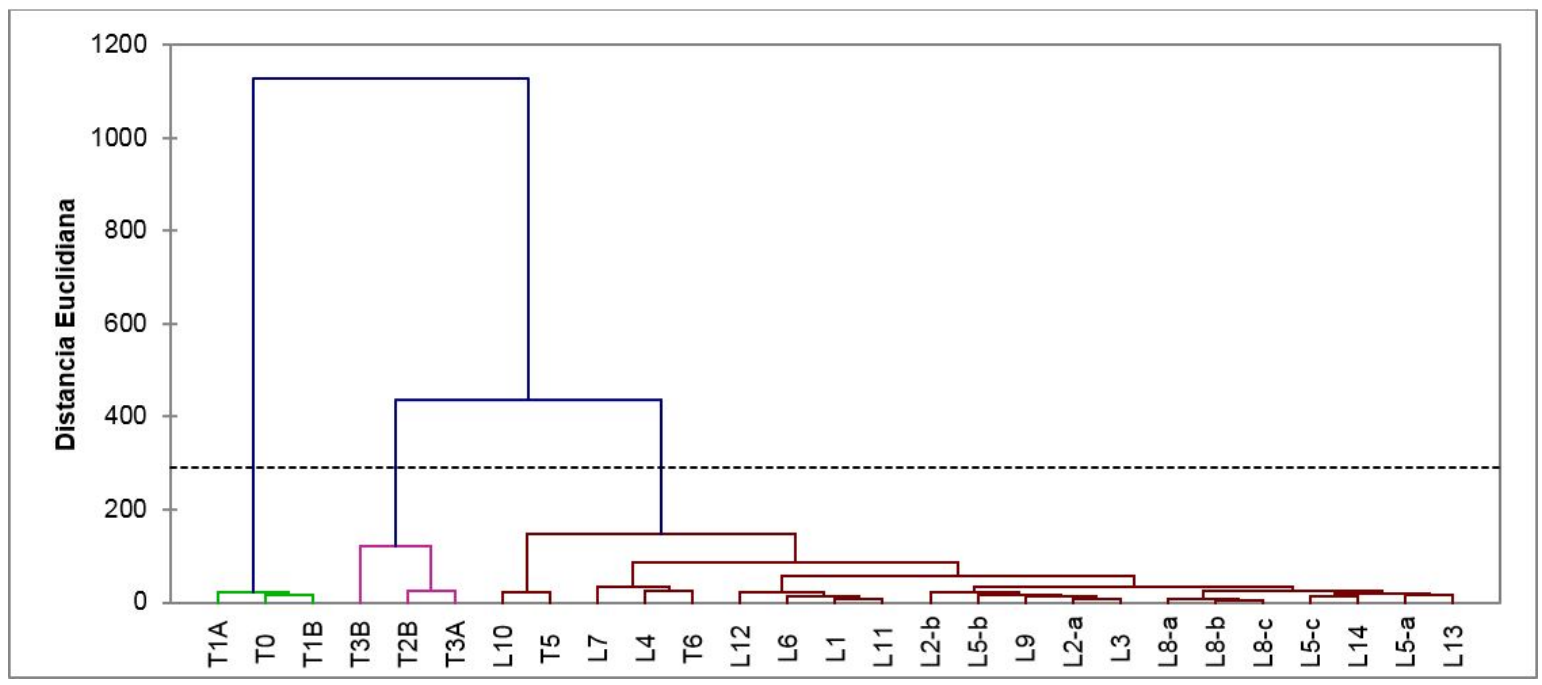

Figura 2. Cladograma de los sitios muestreados en época seca, en función de sus datos abióticos (método aglomerativo, enlace completo, basado en distancias euclidianas).

Para la época de lluvia (Figura 3), la mayoría de los puntos se encuentran en el grupo de L7 a L8-b, correspondientes a 19 sitios en laguna, uno en río (T3B) e igualmente a la salida (T6) y entrada a la laguna (T5), los valores de las variables temperatura, conductividad, oxígeno disuelto y sólidos suspen- didos son bastante similares entre ellos.

El grupo conformado por T2B, T1A y T1B presenta valores similares de $\mathrm{DBO}, \mathrm{DQO}, \mathrm{pH}$ y profundidad. El último grupo (T3A, T0 y T2A) tiene valores similares de oxígeno disuelto, $\mathrm{pH}$ y sólidos suspendidos.

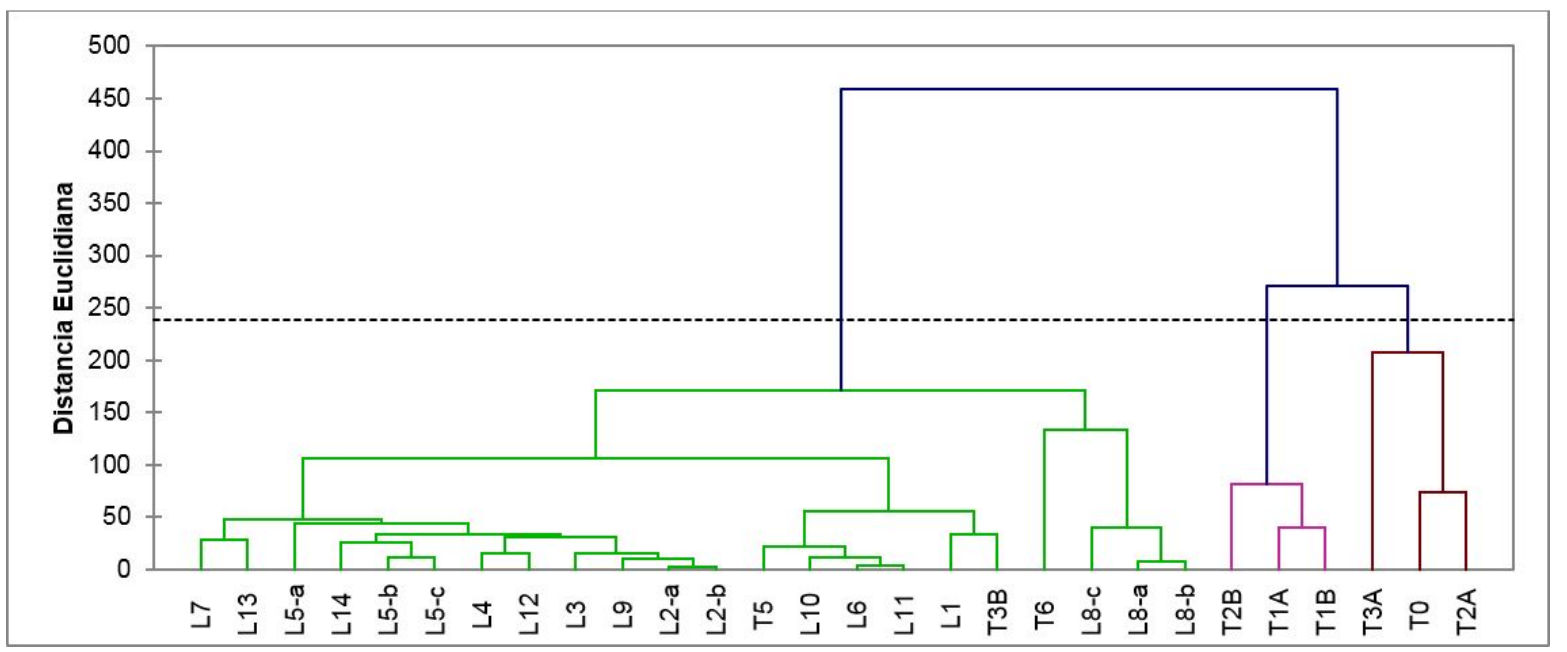

Figura 3. Cladograma de los sitios muestreados en época lluviosa; en función de sus datos abióticos (método aglomerativo, enlace completo, basado en distancias euclidianas). 
3.3 La biota fitoplanctónica y el Índice de Palmer

El resultado de este Índice (IP) fue de $=17$ (Tabla 2); esto es un indicio de carga orgánica media (valores de 15-19 muestran evidencia indirecta de probable contaminación orgánica, según (Taylor et al., 1979). Los géneros presentes en laguna y río en su mayoría son los mismos en las tres épocas de muestreo.

Tabla 2. Índice de Palmer para el fitoplancton registrado, con datos acumulados de las tres épocas de muestreo

\begin{tabular}{cccc}
\hline $\begin{array}{c}\text { Géneros en } \\
\text { Papallacta }\end{array}$ & $\begin{array}{c}\text { Valor del Índice } \\
\text { (Laguna+Río) }\end{array}$ & $\begin{array}{c}\text { Géneros en } \\
\text { Papallacta }\end{array}$ & $\begin{array}{c}\text { Valor del Índice } \\
\text { (Laguna+Río) }\end{array}$ \\
\hline Ankistrodesmus & 2 & Neidium & 0 \\
Campylodiscus & 0 & Nitzschia & 3 \\
Cyclotella & 1 & Oscillatoria & 5 \\
Cymbella & 0 & Pinnularia & 0 \\
Diploneis & 0 & Rhizoclonium & 0 \\
Frustulia & 0 & Rhopalodia & 0 \\
Hantzschia & 0 & Spirogyra & 0 \\
Melosira & 1 & Spirulina & 0 \\
Microcystis & 0 & Surirella & 0 \\
Mougeotia & 0 & Synedra & 2 \\
Navicula & 3 & Total IP & 17 \\
\hline
\end{tabular}

\subsection{Análisis de clasificación de los sitios en función de sus características fitoplanc- tónicas}

En la época seca (Figura 4), los grupos de sitios más relevantes fueron:

El más grande (T3A a L2-b), compuesto por ocho sitios de laguna y cuatro de río (T3A, T5, T2A y T1A); este grupo presenta valores similares de abundancia de Melosira, Navicula, Nitzschia, Synedra, Microcystis, Oscillatoria y Hantzschia. Un segundo conformado por ocho sitios, siete de laguna (L4 a L9) y uno de río (T2B), con los siguientes géneros preponderantes: Navicula, Neidium, Nitzschia, Synedra y Oscillatoria.

Un tercer grupo, conformado por cinco sitios (L1, L13, T6, L11 y T0) con tres sitios de laguna, un efluente de ésta (T6) y un punto en río (T0), con abundancias similares de Melosira, Hantzschia, Navicula, Nitzschia, Synedra, Rhizoclonium y Cyclotella.

Finalmente, L6 en cambio se muestra separado debido a su mayor abundancia de Cyclotella y Rhopadolia. Mientras que dos sitios de río (T1B y T3B) registran abundancias mayores de Campylodiscus, Pinnularia y Mougeotia, ausentes en los otros sitios. 


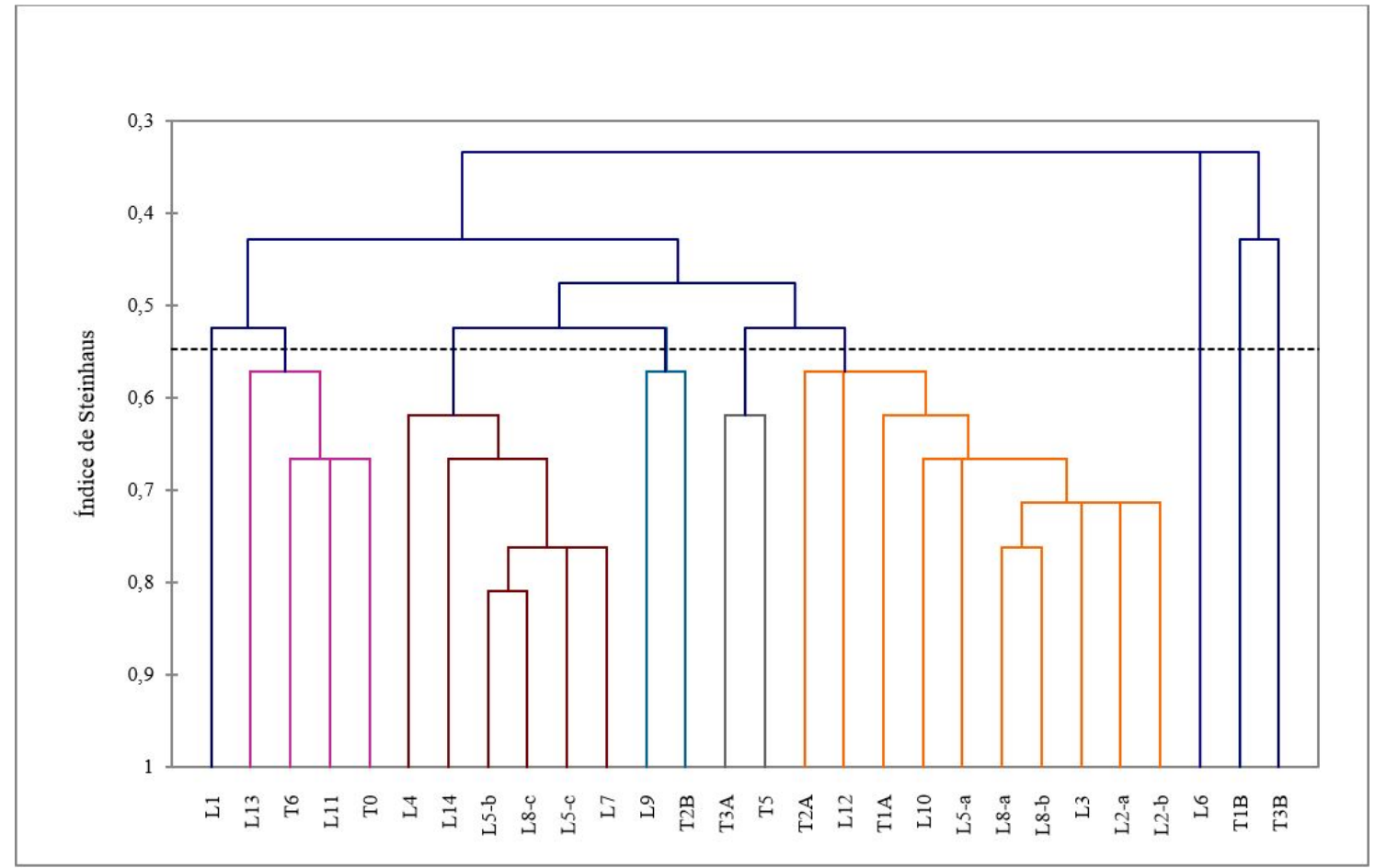

Figura 4. Cladograma de los sitios muestreados en época seca; en función de sus datos fitoplanctónicos (método aglomerativo, enlace completo, basado en el Índice de Steinhaus).

Para la época de lluvia (Figura 5) se observan tres grupos mejor definidos:

El más grande (T5 a L8-b) conformado solo por sitios de laguna (L) más un afluente de ésta (T5), caracterizado por la mayor abundancia de Navicula, Nitzschia y Synedra.

El segundo grupo (T1A a T0), conformado ma- yormente por sitios de río, más dos de laguna (L2-b y L11) y un efluente de laguna (T6); en ellos predominaron Melosira, Cymbella, Navicula, Nitzschia, Synedra y Oscillatoria.

Finalmente, el último grupo conformado por dos puntos de río (T1B y T3B), y con abundancias similares de Navicula y Spirulina. 


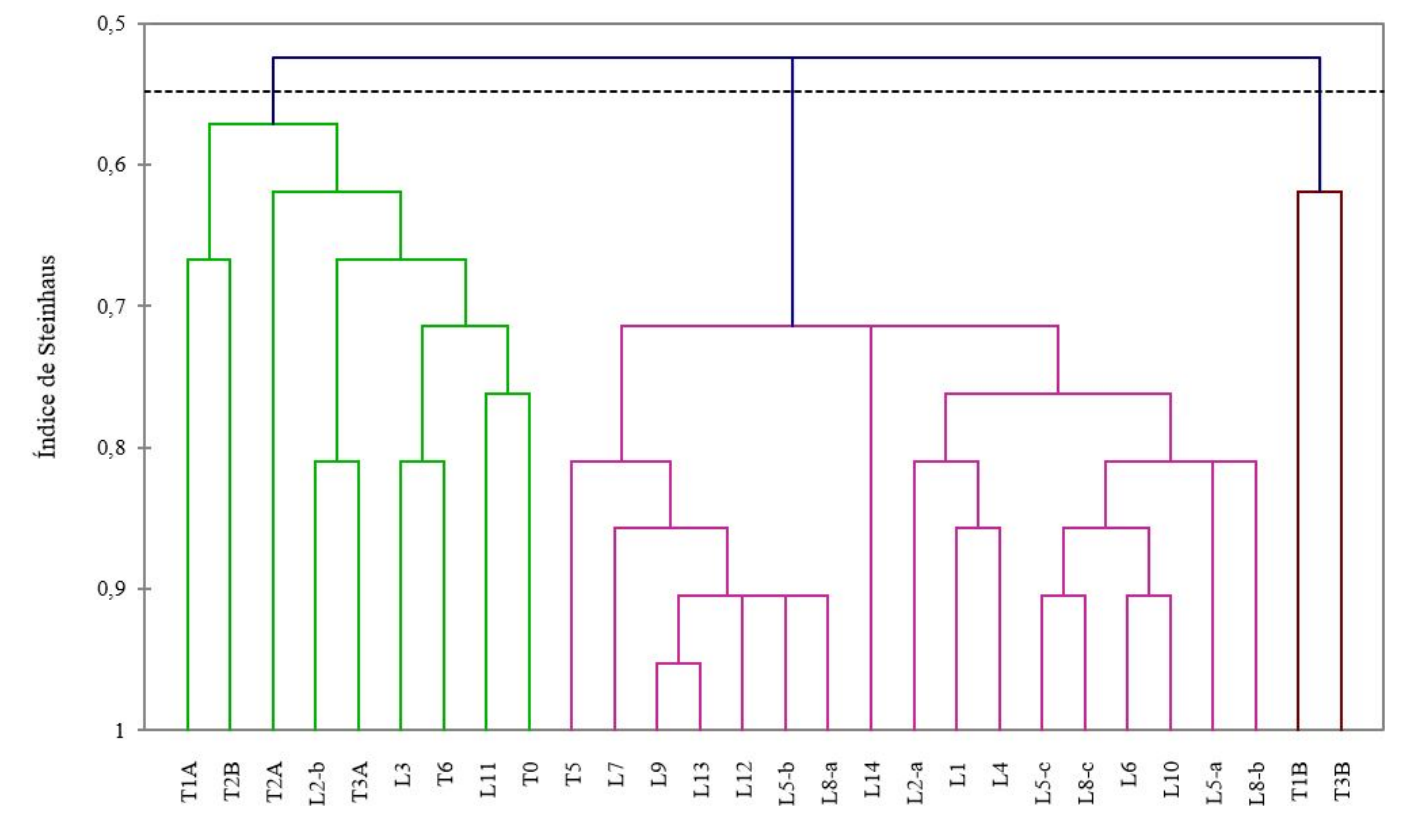

Figura 5. Cladograma de los sitios muestreados en época lluviosa; en función de sus datos fitoplanctónicos (método aglomerativo, enlace completo, basado en el Î́ndice de Steinhaus).

\subsection{Análisis Canónico de Corresponden- cias}

Los parámetros más influyentes que definieron la mayor presencia y abundancia de fitoplancton en los sitios de muestreo fueron: la temperatura superficial del agua (TEMP SUP) y la conductividad (COND), los sólidos suspendidos (SOLD), la trans- parencia del agua determinada por Disco Secchi (TRANSP), la profundidad de la columna de agua (PROF) y finalmente la DBO y DQO. Solo estas variables abióticas se muestran como vectores en las Figuras 6 y 7, las demás variables generaron vectores de muy poca longitud por lo cual no son graficadas. 


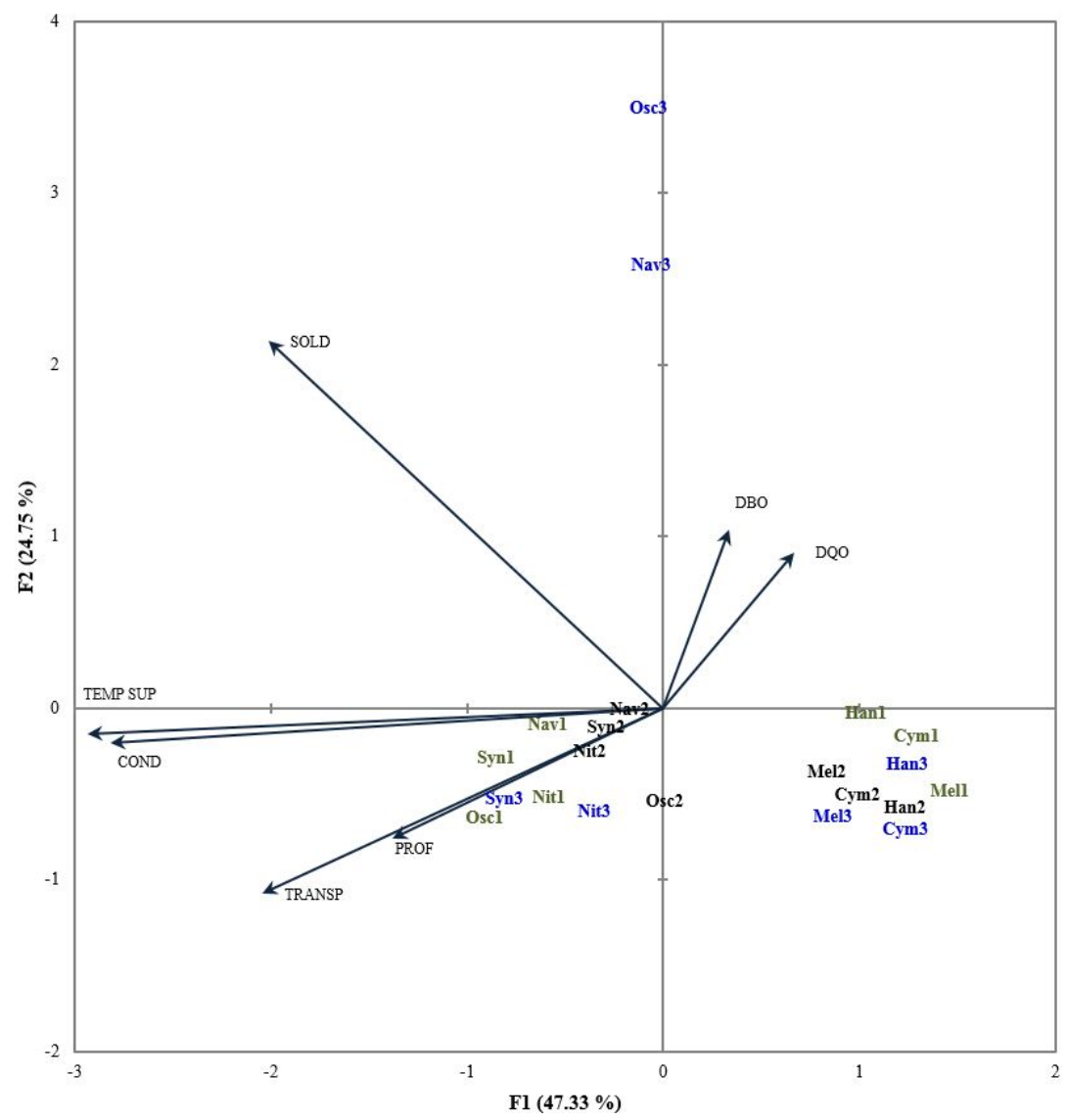

Figura 6. Plano de ordenamiento de géneros de fitoplancton (signados solo con sus tres primeras letras) en relación a variables físico-químicas del agua, generado por un Análisis Canónico de Correspondencias, con datos de las tres épocas de muestreo: en verde los géneros en la época seca acompañados por el número 1; en negro, los mismos en la época de transición con el número 2, y en azul en la época lluviosa con el número 3.

Los géneros que dependen o están en correlación con mayores valores de temperatura superficial, conductividad, transparencia y profundidad total son Synedra (Syn) y Nitzschia (Nit) durante las tres épocas muestreadas (Figura 6). Navicula (Nav) y Oscillatoria (Osc) igualmente están influenciados por estas variables; sin embargo, durante la época de lluvia, ambas se alejan de este grupo y al parecer en esta época su presencia se ve influenciada más por los sólidos suspendidos. Es interesante como Oscillatoria durante la época de transición se aleja de los vectores, lo que puede indicar una poca influencia de las variables abióticas en este género.

El grupo conformado por Melosira (Mel), Cymbella (Cym) y Hantzschia (Han) parecen no depender de ninguna variable abiótica en específico, durante las tres estaciones, muestran una correlación negativa ante las variables.
Analizando las variables abióticas en relación a los sitios de muestreo (Figura 7) se puede observar una distribución consistente: los sitios de laguna (L) están agrupados de manera cercana entre sí, con valores similares de temperatura superficial, conductividad, profundidad total y transparencia del agua. T0, T3A, T6, T5 y T2B (puntos de río) también se encuentran asociados a estos mismos vectores, lo cual indica una influencia importante de estas variables sobre ellos; cabe resaltar que los géneros Synedra, Navicula yNitzschia presentan altos valores de abundancia en los sitios mencionados.

El sitio T2A no tiene una relación clara con la mayoría de variables, excepto con Sólidos Suspendidos, lo cual coincide también con una mayor distribución de Navicula y Oscillatoria en la época de lluvia, es importante mencionar que las abundancias de estos dos géneros durante esta estación pre- 
sentan los valores más altos en este punto.

El último grupo se encuentra conformado por T1B y T1A, que muestran valores bajos de todas las variables abióticas, esto coincide con mayor presencia y abundancia de Melosira, Cymbella y Hantzschia en estos puntos.

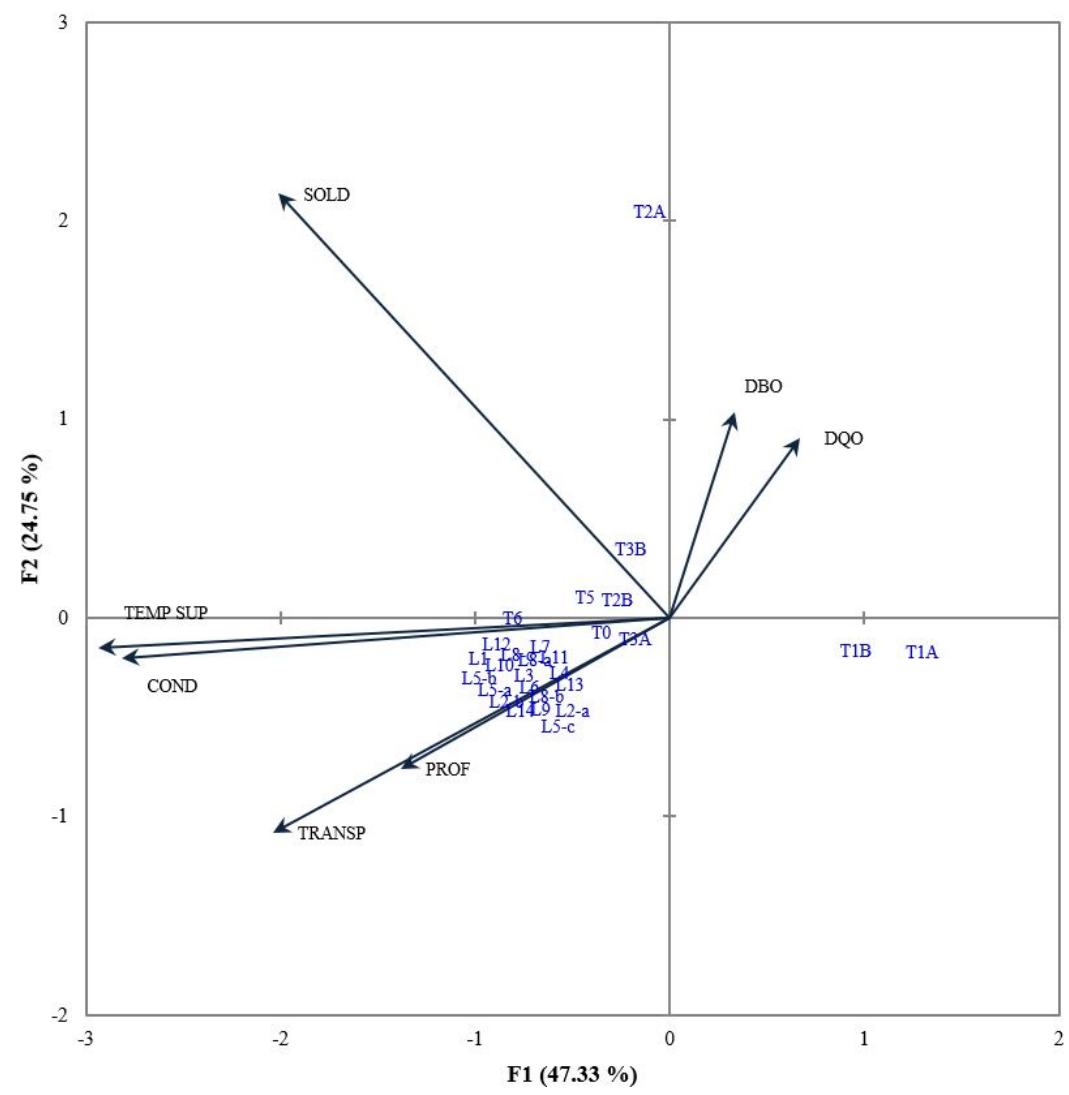

Figura 7. Plano de ordenamiento de los sitios de muestreo en relación a las variables físico-químicas del agua, generado por un Análisis Canónico de Correspondencias, con datos de las tres épocas de muestreo: los sitios codificados en color azul (L= sitios en laguna; $\mathrm{T}=$ sitios en río).

\subsection{Consideraciones ecológicas fundamen- tales}

Se puede mencionar que hacia 2011-2012 (8-9 años después del derrame) existe un estado mesotrófico en la Laguna de Papallacta y sus afluentes, esto se determina debido a la presencia de varios grupos bioindicadores como Navicula, Nitzschia y Oscillatoria, que indican que hay concentración de carga orgánica moderada, pero no determinan alta eutrofización. Este estado actual puede deberse a diferentes causas antrópicas, como descargas de agroquímicos, detergentes, y posibles residuos del derrame de petróleo de 2003.

Es también importante destacar que ningún gé- nero de fitoplancton encontrado tiene una dependencia estricta hacia alguna variable abiótica determinada; más bien, dependen de manera simultánea de varias, corroborando lo propuesto por (Yin, Zheng y Song, 2011).

La presencia y abundancia de géneros de fitoplancton se encontraría determinada por varios procesos del ecosistema (Reynolds, 2006), lo cual implica alguna variación estacional o no de las variables abióticas. Por ejemplo, en la época lluviosa, se registraron las abundancias más bajas de fitoplancton en la mayoría de géneros, excepto en $\mathrm{Me}$ losira, Hantzschia, Navicula, Nitzschia y Synedra, los cuales tienen una presencia relativamente constante en las tres épocas, tanto en río como en laguna. 
La presencia de grupos fitoplanctónicos en la zona fótica de la laguna y sobre todo en los ríos indica que hay buenas condiciones para el desarrollo de producción primaria, incluso con los disturbios a los que ha estado expuesta la zona de estudio.

\subsection{Consideraciones en torno a las varia- bles abióticas}

A pesar de que pequeños cambios de las variables abióticas analizadas no conllevan a una variación importante en la presencia y abundancia de fitoplancton, se considera que la existencia de variaciones mayores ocasionaría una modificación importante en la estructura de la comunidad fitoplanctónica local.

Por ejemplo, la temperatura resulta determinante para la reproducción de los organismos planctónicos, debido a que influye directamente sobre su fisiología y en la físico-química del ambiente acuático (González-Dávila, 1995; Aznar Jiménez, 2000; Bahnasawy, Khidr y Dheina, 2011); en este sentido, cabe mencionar que en Papallacta durante la época seca se registraron los valores más altos de temperatura del agua $\left(6-10^{\circ} \mathrm{C}\right)$ al igual que la mayor riqueza y abundancia de organismos; por lo que es muy probable que esta variable sea determinante en ésta y otras lagunas andinas (Olguín et al., 2004; Manjare, Vhanalakar y Muley, 2010).

Sin embargo, se deben considerar el resto de variables que pueden tener también influencia sobre los organismos planctónicos, en especial debido a que la temperatura se correlaciona (positiva o negativamente) con factores como el $\mathrm{pH}$, sólidos suspendidos, concentración de oxígeno y conductividad (Aznar Jiménez, 2000; Anukool y Shivani, 2011); esta última en Papallacta presentó valores menores durante la época de lluvia (entre $80 \mu \mathrm{S}$ y $400 \mu \mathrm{S}$ ) en relación a la época seca y de transición en las que se registraron valores entre $120 \mu \mathrm{S}$ y $800 \mu \mathrm{S}$. Estos valores elevados de conductividad son poco usuales en la mayoría de fuentes de agua de los ecosistemas de páramo. En Papallacta, sin embargo, se tiene un aporte de aguas termales de origen volcánico que se descargan en los ríos afluentes de la laguna, lo cual explicaría tales valores.

Un patrón similar se observa con los sólidos suspendidos, los cuales durante la estación lluviosa muestran valores bajos, coincidiendo con un aumento en la turbidez durante esta estación, además el paso de luz natural llega a menor profundidad
$(0,5-0,9 \mathrm{~m})$, a diferencia de la época seca y de transición, en las cuales los valores de transparencia alcanzaron mayor profundidad (1-5 m). Este mayor o menor paso de luz en la columna de agua de la laguna resulta ser otro factor importante que afecta directamente al comportamiento de los organismos, por ser vital para la fotosíntesis, tal como lo mencionan Reynolds (2006); Belinger y Sigee (2010).

Esta incidencia de luz, que depende de la turbidez y los sólidos en suspensión, puede ser determinante sobre la riqueza y abundancia de los géneros de fitoplancton, porque en época seca y transición (con valores de turbidez bajos y mayor paso de luz) se registraron mayor riqueza y abundancia de fitoplancton en relación a la época de lluvia, en la que se registraron valores menores (con turbidez alta y menor transparencia).

Los valores de oxígeno disuelto fueron relativamente constantes en las tres épocas, a excepción de unos pocos sitios en época lluviosa. El pH se mantuvo entre siete y nueve, las variaciones dentro de este rango no influyen directamente sobre algún organismo particular de fitoplancton. Para DBO y DQO se registran valores altos durante la época de lluvia, especialmente el $\mathrm{DBO}$ ya que estos altos valores coinciden con la baja abundancia de fitoplancton, que son los encargados de descomponer la materia orgánica que representa esta variable.

Los valores de nitrógeno total son relativamente bajos en todas las campañas ( 0 a $3 \mathrm{mg} / \mathrm{l}$ ), éste es un componente limitante de crecimiento para algunos grupos de fitoplancton (Collos y Berges, 2011); las cianobacterias, por ejemplo, son importantes fijadoras de nitrógeno y su presencia en la laguna y ríos muestreados es limitada, lo cual se puede vincular a la disponibilidad de nitrógeno.

El aumento del nivel del agua en la época de lluvia sin duda afecta a diferentes variables abióticas, ya que se diluyen las concentraciones de sólidos suspendidos y baja la conductividad, las cuales son usualmente altas por la influencia de las aguas termales de la zona, mismas que aportan con importantes cantidades de sales de azufre especialmente en las épocas seca y de transición; los sólidos suspendidos también fueron afectados por esta dilución (valores entre $30 \mathrm{mg} / 1$ y $240 \mathrm{mg} / 1$ en época de lluvia, en relación a $70 \mathrm{mg} / 1$ y $560 \mathrm{mg} / 1$ en época seca y de transición). También, debido a las corrientes y el viento propios de la época lluviosa hay una remoción de sólidos desde las orillas, distribuyéndose homogéneamente sobre toda la superficie de la la- 
guna, aumentando la turbidez y limitando el paso de luz.

\subsection{Relación de la presencia de géneros fi- toplanctónicos con la estacionalidad}

El estado mesotrófico del sistema lacustre en general, como ya se mencionó, se ha basado en la presencia y abundancia de ciertos géneros fitoplanctónicos. Por ejemplo, existen cinco géneros de diatomeas en todas las épocas muestreadas, a la vez son los más abundantes tanto en laguna como en río: Melosira, Hantzschia, Navicula, Nitzschia y Synedra; además, otros géneros también se presentaron recurrentemente pero con menores valores de abundancia: Cymbella, Oscillatoria, Microcystis y Ankistrodesmus.

Asimismo, cabe mencionar también que las cianobacterias y algas verdes descritas son comunes en aguas que presentan contaminación orgánica, y algunas como Ankistrodesmus incluso pueden causar contaminación luego de su presencia; considerándose a este grupo como un contaminante natural capaz de afectar la concentración de oxígeno (Belinger y Sigee, 2010; Likens, 2010).

En general, los resultados indican que el ecosistema estudiado reúne las condiciones necesarias para que existan tipos y formas de vida fitoplanctónicos diversos, aún después del derrame de petróleo de 2003, permitiendo a su vez que se desarrollen otros organismos de niveles tróficos superiores (zooplancton, macroinvertebrados acuáticos, anfibios, peces).

\section{Conclusiones}

Debido a la naturaleza del fitoplancton que se encuentra suspendido en la columna de agua, a su dependencia de las corrientes para desplazarse y su metabolismo, se consideran organismos capaces de absorber metales pesados de forma relativamente rápida; este fenómeno pudo haber ocurrido en Papallacta: una absorción parcial pasiva por parte del fitoplancton de algunos hidrocarburos disueltos, mediante el intercambio de iones directamente hacia la pared celular.

Posiblemente el fitoplancton en Papallacta absorbió algunos hidrocarburos durante los primeros días después del derrame, las repercusiones inmediatas pudieron haber sido una rápida disminución de estos organismos en las zonas afectadas y cambios en su metabolismo.

Sin embargo, cuando ya el crudo se precipitó hacia el fondo de la laguna, la comunidad fitoplanctónica se habría recuperado bastante bien y continuado un desarrollo regular en la superficie (hasta unos cinco a seis metros de profundidad), habiéndose inclusive llegado a obtener para 2012 una calidad fitoplanctónica similar a la reportada por Kannan (1979), previa al derrame de petróleo. Esto se corrobora debido a que varios géneros encontrados en 1979, como Synedra y Melosira, ya se estaban presentes en 2011 mostrando cierta abundancia; otros como Pinnularia y Nitzschia, también descritos en ese estudio, aún están presentes. Fragilaria y Ampho$r a$ son dos géneros encontrados en 1979 y que en el presente estudio no fueron observados, por lo que es posible que hayan desaparecido a consecuencia del derrame.

Debido a que el crudo se precipitó hacia el fondo de la laguna, posiblemente sea allí donde pudiera todavía haber contaminación muy focalizada por TPHs (Hidrocarburos Totales de Petróleo). La misma tendencia se puede observar en los ríos afluentes, ya que el crudo, debido a las corrientes, se depositó en la laguna, lo cual pudo ayudar a que el ecosistema ripario se recupere más rápido (autodepuración); hacia 2011 prácticamente ya no existía presencia de crudo allí (en todos los puntos de río los TPH están entre $0.3 \mathrm{mg} / 1$ a $0.26 \mathrm{mg} / \mathrm{l}$ ).

En el caso de los ríos locales, ya que no existen estudios de las comunidades fitoplanctónicas previos al derrame, no se puede afirmar cuáles géneros estuvieron presentes décadas atrás; sin embargo, se pudiera especular sobre una tendencia similar en el pasado sobre la composición y abundancia de fitoplancton en los ríos afluentes: una recuperación en los últimos años. Esta afirmación se basa en que como el río alimenta a la laguna, mucho del fitoplancton lacustre llega desde sus afluentes, y a pesar de que ambos ecosistemas tienen dinámicas distintas y el fitoplancton pudiera tener formas de vida diferentes, los mismos géneros están presentes en ambos ecosistemas.

El enunciado anterior se ve consolidado también debido a la naturaleza polimíctica de la Laguna de Papallacta (no presenta estratificación termal por largos períodos y sus aguas se mezclan desde la superficie hacia el fondo) y debido a que es un sistema lacustre abierto (Terneus, 2002; Kannan, 2006; Roldán, 2008). 
Cabe finalmente mencionar que la zona fótica de la laguna tiene desde 2012 las condiciones para albergar vida a pesar de los cambios ambientales estacionales y del derrame de petróleo ocurrido. Aun así sería recomendable monitorear este sistema hídrico cada cuatro o cinco años, y observar a largo plazo su comportamiento sucesional.

\section{Agradecimientos}

A la Empresa Estatal de Petróleos PETROECUADOR, la cual a través de ESINGECO Cía. Ltda., financiaron el presente Proyecto. A la Escuela de Biología de la Universidad Internacional del Ecuador UIDE, por brindar las facilidades en los laboratorios para la identificación taxonómica y conteo de los especímenes. A María de Lourdes Guerra, por su apoyo en el trabajo taxonómico.

\section{Referencias}

Alberta Environment. 2006. Aquatic Ecosystems Field Sampling Protocols. Alberta Environment.

Anukool, Srivastava y Srivastava Shivani. 2011. "Assessment of Physico-Chemical properties and sewage pollution indicator bacteria in surface water of River Gomti in Uttar Pradesh." International Journal of Environmental Sciences 2(1):325.

Arce, O, R Herbas, F Rivero y A. González. 2006. "Indicadores biológicos de calidad del agua." Universidad Mayor de San Simón. Cochabamba, Bolivia. Online: https://goo.gl/K4oTGB.

Armisen, P, J Cruz y C. Larrosa. 2005. Percepción social del riesgo y vulnerabilidad de la población de Papallacta por derrames petroleros. In XXXIII Curso Internacional de Geografía Aplicada: Geografía y Riesgos Ambientales. CEPEIGE, Quito.

Aznar Jiménez, Antonio. 2000. “Determinación de los parámetros físico-químicos de calidad de las aguas." Revista interdisciplinar de gestión ambiental 2(23):12-19.

Bahnasawy, Mohamed, Abdel Aziz Khidr y Nadia Dheina. 2011. "Assessment of heavy metal concentrations in water, plankton, and fish of Lake Manzala, Egypt." Turkish Journal of Zoology 35(2):271-280.
Belinger, EG y DC Sigee. 2010. "Freshwater Algae: Identification and use as bioindicators. published by John Wiley and Sons.".

California Academy of Sciences. 2012. “Catalogue of Diatom Names.".

Canter-Lund, H. 2012. "The Hilda Canter-Lund Fresh Water Algae Collection.".

Collos, Y y J. Berges. 2011. “Nitrogen Metabolism in Phytoplankton." Mar. Ecol., 1(1).

Delgado-Molina, Jose Antonio, Presentación Carrillo, Juan Manuel Medina-Sánchez, Manuel VillarArgaiz y Francisco José Bullejos. 2009. “Interactive effects of phosphorus loads and ambient ultraviolet radiation on the algal community in a high-mountain lake." Journal of plankton research 31(6):619-634.

Escobar, María J, Esteban Terneus y Patricio Yánez. 2013. "El plancton como bioindicador de la calidad del agua en zonas agrícolas andinas, análisis de caso." Qualitas 5(1):17-37.

Fourtanier, Elisabeth y John Patrick Kociolek. 2009. Catalogue of diatom names. California Academy of Sciences San Francisco, CA.

González-Dávila, Melchor. 1995. "The role of phytoplankton cells on the control of heavy metal concentration in seawater." Marine Chemistry 48(34):215-236.

Goslee, Sarah C, Dean L Urban et al. 2007. "The ecodist package for dissimilarity-based analysis of ecological data." Journal of Statistical Software 22(7):1-19.

Hern, SC, Llewellyn R Williams, William Dee Taylor, Victor W Lambou y MK Morris. 1979. Phytoplankton water quality relationships in US lakes. Environmental Protection Agency, Office of Research and Development,[Office of Monitoring and Technical Support], Environmental Monitoring and Support Laboratory.

Hill, Thomas, Pawel Lewicki y Paweł Lewicki. 2006. Statistics: methods and applications: a comprehensive reference for science, industry, and data mining. StatSoft, Inc.

Kannan, M. S. 2006. "Informe para Ecuambiente sobre el monitoreo de la laguna de Papallacta efectuado el 25 de julio de 2006.". 
Kannan, Miriam Steinitz. 1979. Comparative limnology of Ecuadorian lakes: a study of species number and composition of plankton communities of the Galapagos Islands and the Equatorial Andes $\mathrm{PhD}$ thesis The Ohio State University.

Likens, Gene E. 2010. Plankton of inland waters. Academic Press.

Manjare, SA, SA Vhanalakar y DV Muley. 2010. "Analysis of water quality using physicochemical parameters Tamdalge tank in Kolhapur district, Maharashtra." International Journal of Advanced Biotechnology and Research 1(2):115-119.

Marshall, Harold G. 1965. "The annual distribution and stratification of phytoplankton at Aurora Lake, Portage County, Ohio." Ohio Journal of Science 65(4)).

Molina Santos, Marcos Gonzalo. 2013. Efectos del derrame de petróleo sobre la comunidad Fitoplanctónica de la laguna de Papallacta y sus principales afluentes. B.S. thesis Quito/UIDE/2013.

Olguín, Héctor F, Alba Puig, Carolina R Loez, Alfredo Salibián, Mirta L Topalián, Patricia M Castañé y María G Rovedatti. 2004. “An integration of water physicochemistry, algal bioassays, phytoplankton, and zooplankton for ecotoxicological assessment in a highly polluted lowland river." Water, air, and soil pollution 155(1-4):355-381.

O'Sullivan, Patrick y Colin Reynolds. 2004. “The Lakes Handbook, Volume 1: Limnology and Limnetic Ecology." Limnology 5:125-126.

PetroEcuador. 2006. "Examen de control ambiental al derrame de crudo ocurrido en el área de la Reserva Cayambe-Coca por la ruptura de la tubería del Sistema del Oleoducto Transecuatoriano (SOTE), a cargo de Petroecuador.".

Pino, Iván A Aveiga del, Luis Cabrera, Iván Tapia y Patricio Ponce. 2005. Remediación de los impactos causados por el derrame de petróleo en la laguna de papallacta en ecuador-sur america tiempo de ejecución: noviembre 2003-mayo 2004. In International Oil Spill Conference. Vol. 2005 American Petroleum Institute pp. 1035-1038.

PRAA. 2007. “Diseño e Implementación de Medidas Piloto de Adaptación al Cambio Climático en la Región Andina (PRAA/Ecuador).".
Reynolds, Colin S. 2006. The ecology of phytoplankton. Cambridge University Press.

Roldán, G., \& Ramírez J. 2008. "Fundamentos de Limnología Neotropical." Editorial Universidad de Antioquia .

Suthers, Iain M y David Rissik. 2009. Plankton: A guide to their ecology and monitoring for water quality. CSIRO publishing.

Taylor, W, L Williams, S Hern, Morris F Lambou, V and. y M. Morris. 1979. "Phytoplankton water quality relationships in U.S. Lakes. Disponible en: http:/ / xurl.es/izokh." Environmental Protection Agency. Las Vegas, USA p. 220. Disponible en: http://xurl.es/izokh.

Ter Braak, Cajo JF y Piet FM Verdonschot. 1995. "Canonical correspondence analysis and related multivariate methods in aquatic ecology." Aquatic sciences 57(3):255-289.

Terneus, E. 2002. Estructura y composición florística de las plantas acuáticas en 70 lagunas de páramo y dos lagunas interandinas del Ecuador PhD thesis Tesis doctoral. Quito: Pontificia Universidad Católica del Ecuador.

Terneus, Esteban. 2018. “Principios fundamentales en torno a la calidad del agua, el uso de bioindicadores acuáticos y la restauración ecológica fluvial en Ecuador." LA GRANJA. Revista de Ciencias de la Vida 27(1):36-50. Online: http:/ /doi.org/10. 17163/lgr.n27.2018.03.

Yánez, P. 2005. "Biometría y Bioestadística fundamentales." Analizando la estructura numérica de la información en proyectos ecológicos .

Yánez, Patricio. 2014. Ecología y biodiversidad: un enfoque desde el neotrópico. Universidad Iberoamericana del Ecuador.

Yin, Dacong, Lingling Zheng y Lirong Song. 2011. "Spatio-temporal distribution of phytoplankton in the Danjiangkou Reservoir, a water source area for the Southto-North Water Diversion Project (Middle Route), China." Chinese Journal of Oceanology and Limnology 29(3):531-540.

Yoshioka, Paul M. 2008. "Misidentification of the Bray-Curtis similarity index." Marine Ecology Progress Series 368:309-310. 